\title{
Bragg Rods and Multiple X-Ray Scattering in Random-Stacking Colloidal Crystals
}

\author{
A.V. Petukhov, ${ }^{1, *}$ I. P. Dolbnya, ${ }^{2}$ D. G. A. L. Aarts, ${ }^{1}$ G. J. Vroege, ${ }^{1}$ and H. N.W. Lekkerkerker ${ }^{1}$ \\ ${ }^{1}$ van 't Hoff Laboratory for Physical and Colloid Chemistry, Debye Institute, University of Utrecht, \\ Padualaan 8, 3508 TB Utrecht, The Netherlands \\ ${ }^{2}$ DUBBLE CRG/ESRF, The Netherlands Organisation for Scientific Research, ESRF BP220, F-38043, Grenoble Cedex, France
}

(Received 15 May 2002; published 17 January 2003)

\begin{abstract}
Synchrotron small-angle $\mathrm{x}$-ray diffraction images of random-stacking-induced Bragg scattering rods are obtained in a wide range of wave vectors from a single colloidal crystal. The results reveal a strong multiple scattering effect, which leads to new features in the diffraction pattern - secondary Bragg rods. We argue that dynamic $\mathrm{x}$-ray diffraction is rather common for high-quality colloidal photonic crystals and should be taken into account.
\end{abstract}

The spontaneous formation of crystals in dispersions of colloidal spheres $[1,2]$ remains an intriguing phenomenon, which has aroused renewed interest because of its photonic applications [3]. Modern microscopy [4-7] allows 3D imaging of colloidal crystals giving mainly information on local structure and ordering. Scattering techniques are more appropriate for the study of longrange order in colloidal crystals. Until recently this involved primarily light scattering [2,8-11], which limits the range of scattering vectors severely and is known to be extremely prone to multiple scattering. In the $\mathrm{x}$-ray field a new era has spawned by the advent of synchrotron radiation, which offers high-quality $\mathrm{x}$-ray beams and has led to the development of 2D x-ray detectors. These now permit small-angle $\mathrm{x}$-ray diffraction (SAXD) on colloidal single crystals [12] and allow us to achieve a resolution sufficient to determine long-range order parameters in high-quality crystals [13]. So far, the so-called kinematic theory, where it is assumed that diffraction is weak, has been used [12-15] to describe x-ray diffraction patterns from colloidal crystals. However, already long ago it was realized that in perfect crystals with long-range periodic positional order, the kinematic approach fails and one has to use the more complicated dynamic theory [16], which accounts for mutual interactions between the incident and all the diffracted waves. While for SAXD in colloidal crystals the applicability of the kinematic approach is hardly ever questioned, our results indicate a significant effect of dynamic $\mathrm{x}$-ray diffraction, which reveals itself in our crystals in an unusual way as novel features in the diffraction pattern.

Calculations [17] indicate that colloidal hard spheres should favor a face centered cubic crystal as a closepacked structure, but in practice a random hexagonal close-packing (rhcp) structure is rather more common $[5,9-11,13,14]$ either as a transient state or possibly even permanently (factors such as polydispersity may readily influence the very small free energy differences involved). Because of the irregular stacking of hexagonal layers in three possible lateral positions, some features in the reciprocal lattice of rhcp are smeared out into Bragg scattering rods $[10,13,14,18]$ in the direction perpendicular to the hexagonal layers [Fig. 1(a)]. Periodicities common to all layers lead to sharp Bragg spots.

We have been able to image Bragg scattering rods directly in a single diffraction pattern within a wide range of diffraction wave vectors. Moreover, the combination of our crystals, showing long-range spatial order [13], and the high-quality synchrotron beam allow us to observe multiple scattering in a spectacular way. Because of the random stacking, multiple scattering here leads to qualitatively different features (secondary Bragg rods) opposed to a mere change in intensities of spots expected for ordinary crystals with full 3D periodicity.

The same set of samples and a similar $\mathrm{x}$-ray diffraction setup as those described in [13] were used. In brief, samples were prepared by filling flat glass capillaries with dilute colloid/polymer suspensions with 5\% volume fraction of sterically stabilized silica hard spheres [19] of radius $R=112 \mathrm{~nm}$; effectively about $10 \%-15 \%$ of the volume was filled by penetrable random polymer coils of $14 \mathrm{~nm}$ radius of gyration. The polymer induces a weak
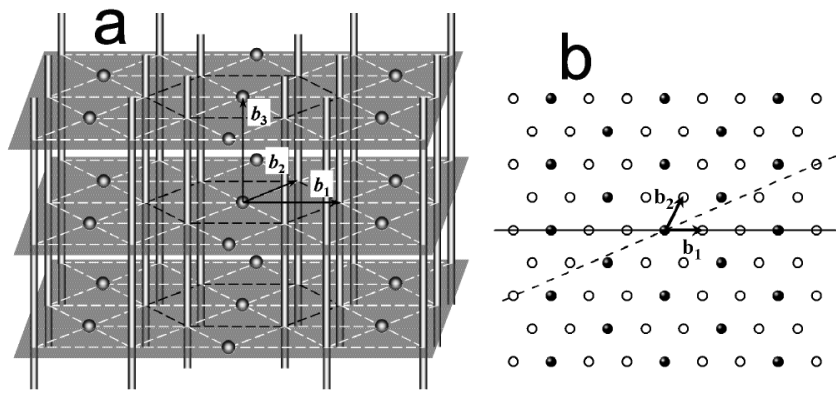

FIG. 1. (a) Sketch of the reciprocal lattice of randomly stacked hexagonal close-packed crystals. The shadowed planes are to guide the eye. (b) Top view of the reciprocal lattice illustrating the orientation of the Ewald sphere in Fig. 2 (solid line) and Fig. 3 (dashed line). The closed symbols correspond to lines of localized reflections (spots) while the open symbols display the position of rods. 
depletion attraction between the silica spheres promoting spontaneous formation of large single crystals [13] (up to about $1 \mathrm{~mm}$ along the capillary walls and usually filling the $0.2 \mathrm{~mm}$ space between the flat walls of the capillary allowing for single-crystal diffraction measurements). The experiments were performed about 18 months after crystallization at the BM26 "DUBBLE" beam line at the European synchrotron radiation facility in Grenoble, France. Diffraction induced by an $\mathrm{x}$-ray beam with wavelength $\lambda=1.24 \AA$ was recorded at $8 \mathrm{~m}$ distance from the sample.

The diffraction wave vector $\mathbf{q}=\mathbf{k}_{0}-\mathbf{k}$ must lie on the so-called Ewald sphere since the wave vectors, $\mathbf{k}_{0}$ and $\mathbf{k}$, of the incident and diffracted waves have the same length of $2 \pi / \lambda$. For colloidal crystals $q \ll k_{0}$ so that diffraction is observed only at small angles and the diffraction vector $\mathbf{q}$ is practically normal to $\mathbf{k}_{0}$. The relevant part of the Ewald sphere is then extremely flat [20]. The diffraction vector $\mathbf{q}$ can be written in terms of three basis vectors $\mathbf{q}=h \mathbf{b}_{1}+k \mathbf{b}_{2}+l \mathbf{b}_{3}$ introduced in Fig. 1(a), where $h$ and $k$ are integers due to the in-plane periodicity. Bragg spots are observed for $h-k$ divisible by 3 and integer values of $l$. For Bragg rods $h-k$ is not divisible by 3 and $l$ is any real number.

To directly image the Bragg rods on the detector, the Ewald sphere must produce a vertical cut through the reciprocal lattice of Fig. 1(a). In our flat capillaries one can find crystals with various orientations suggesting that the crystals did not nucleate at the glass wall but rather at the top interface of the concentrated sediment. In our previous work [13] a crystal with hexagonal planes (nearly) parallel to the flat capillary walls was studied. Now we have chosen a crystal with hexagonal planes making an angle of about $60^{\circ}$ with respect to the capillary wall, enabling us to send the incoming $\mathrm{x}$-ray beam parallel to the crystal planes. Careful orientation of the sample leads to a diffraction pattern $I(\mathbf{q})$ as in Fig. 2(a). For this orientation the Ewald sphere cuts through the reciprocal lattice as shown by the solid line in Fig. 1(b). Figure 2(b) shows a magnified view of the structure factor $S(\mathbf{q})=I(\mathbf{q}) / F(q)$, where $F(q)$ is the form factor determined from scattering in a dilute suspension of colloidal particles. X-ray scattering is observed on the detector along many lines, which originate from the Bragg rods of the reciprocal lattice and (each third line) from the localized spots of Fig. 1(a). However, some scattering is also observed in between the spots, which is not expected from the reciprocal lattice [Fig. 1(a)]. As discussed below, this scattering is a fingerprint of dynamic diffraction.

Dynamic diffraction takes place when the interaction of the incident wave with the sample is no longer weak and one has to take into account that the diffracted waves deplete the incident beam and become in turn sources of secondary diffraction. The complexity of the theoretical modeling of such dynamic interactions raises significantly upon increasing the number of mutually interact-
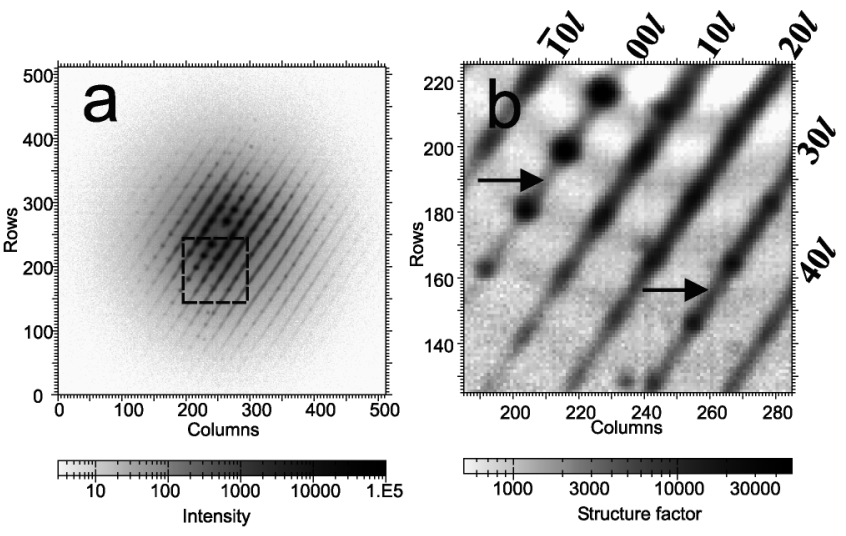

FIG. 2. (a) Diffraction pattern measured for the crystal orientation corresponding to the Ewald sphere position illustrated by the solid line in Fig. 1(b). The direct beam is absorbed by a small beam stop in the middle of the detector. (b) Magnified view of the structure factor $S(\mathbf{q})$ profile within the area marked on panel (a). Arrows point to the secondary Bragg rods in between the sharp Bragg spots.

ing waves [21]. An even more complicated description is developed for visible light waves in photonic materials, where the refractive index contrast is large and the effect of diffraction is not weak even within one period of the structure [22,23]. Almost all theories deal with dynamic diffraction in crystals with full 3D periodicity. The effect of stacking faults lifting periodicity in one direction has been investigated in Ref. [22] and the photonic gap for visible light at normal and grazing incidence is found to broaden. Despite the significant progress of the theories, none of them can be applied to describe our data shown in Fig. 2. Here one has to consider not only multibeam diffraction into many Bragg spots, but also diffraction into a continuum of plane waves (induced via Bragg rods).

To demonstrate that in our system diffraction enters the dynamic regime, one can estimate the strength of diffraction using the much simpler kinematic approach. The power $d P_{\mathrm{sc}}=(d \sigma / d \Omega) I_{0} d \Omega$ of the wave scattered by a single colloidal particle into a small element $d \Omega$ of the solid angle can then be described by the differential scattering cross section $(d \sigma / d \Omega)=r_{0}^{2} Z^{2} F(q)$, where $I_{0}$ is the intensity of the incident wave at the position of the particle, $r_{0}=e^{2} /\left(m c^{2}\right)$ is the Thompson radius, and $Z$ is the excess number of electrons in the colloidal particle relative to an equivalent volume of solvent. The form factor is normalized such that $F(q \rightarrow 0)=1$, and it equals $F(q)=9(\sin q R-q R \cos q R)^{2} /(q R)^{6}$ [24] for a sphere of radius $R$ with a uniform distribution of the electron density. Under the conditions of our experiment (specific weight [19] of silica particles and the solvent cyclohexane are 1.7 and $0.77 \mathrm{~g} / \mathrm{ml}$, respectively, corresponding to a refractive index contrast of $\Delta n=2.1 \times$ $10^{-6}$ for $10 \mathrm{keV} \times$ rays), the total small-angle scattering cross section $\sigma=\int(d \sigma / d \Omega) d \Omega$ of one sphere is about 
$12 \mathrm{~nm}^{2}$, i.e., only $3 \times 10^{-4}$ of its geometrical cross section $\pi R^{2}$. Thus, a single particle only weakly interacts with the $\mathrm{x}$-ray wave.

However, the situation may change drastically if silica spheres form a single crystal possessing long-range order and the incident synchrotron $\mathrm{x}$-ray beam provides conditions for coherent interference on large distances $[13,25]$. If for a sharp $(h k l)$ reflection with $h-k$ divisible by 3 the Bragg condition is fulfilled (i.e., it is crossed by the Ewald sphere), the weak waves scattered by individual spheres interfere constructively and the diffracted power grows quadratically,

$$
P_{(h k l)}=\left(L / L_{(h k l)}\right)^{2} P_{0},
$$

with the distance $L$ traveled by the beam. Here $P_{0}$ is the total power of the incident beam and the characteristic length $L_{(h k l)}$ is determined by [16]

$$
\left(L_{(h k l)}\right)^{-2}=\lambda^{2} n_{\mathrm{sph}}^{2}(d \sigma / d \Omega)_{(h k l)},
$$

where $n_{\text {sph }}$ is the number density of spherical particles. Assuming a close-packed crystal structure with $n_{\text {sph }}=$ $1 /\left(4 \sqrt{2} R^{3}\right)$ and collecting all the numbers in Eq. (2) for the lowest order (001) reflection seen in Fig. 2, one finds $L_{(001)}=0.11 \mathrm{~mm}$, i.e., about half the crystal size along the beam. Clearly, the diffracted power is then comparable to $P_{0}$ and diffraction switches from the kinematic to the dynamic regime. Interestingly, the dependence of $L_{(h k l)}$ on the particle size $R$ cancels in Eq. (2) since $(d \sigma / d \Omega) \propto R^{6}$ and $n_{\text {sph }} \propto R^{-3}$. Thus, for larger spheres dynamic diffraction can be observed for a smaller number of lattice periods. This factor leads to a principal difference between atomic and colloidal crystals in requirements of their perfectness to observe dynamic diffraction. While the former one requires perfect order over $\sim 10^{5}$ lattice constants, in the latter positional order over as little as a few hundreds of lattice periods can break up the kinematic description of $\mathrm{x}$-ray diffraction.

In contrast to a sharp reflection like (001), in a Bragg rod the scattering amplitudes of different hexagonal planes have additional stacking-dependent phase shifts. This significantly reduces the intensity diffracted in one particular direction and spreads the diffraction intensity along the Bragg rod. However, if the crystal possesses long-range in-plane order along the beam, the scattering amplitudes within each layer interfere constructively leading to a similar quadratic dependence of the scattered power with the distance $L$. For example, to evaluate the power $P_{(10 l)}^{[0,1]}$ scattered into a piece of the low-order $(10 l)$ rod between $l=0$ and $l=1$, one has to integrate the form factor $F(q)$ together with the structure factor $S_{\text {rod }}(\mathbf{q})$ arising from interference between contributions of randomly stacked planes. One then finds that within the kinematic theory $P_{(10 l)}^{[0,1]}$ grows as in Eq. (1) with the characteristic length $L_{(10 l)}^{[0,1]}=0.15 \mathrm{~mm}$, i.e., the power scattered into the low-order (10l) rod grows nearly as fast as the power diffracted into the (001) reflection since scattering along the whole rod is possible at this sample orientation. The estimates thus show that the incident $\mathrm{x}$-ray beam is quickly depleted by scattering into the low-order spots and rods, which become in turn sources of strong secondary diffraction. Scattering into the (10l) Bragg rods is able to compete with diffraction into the sharp (001) reflection and the appearance of secondary Bragg rods in Fig. 2 is therefore not surprising.

To reduce the effect of multiple scattering via rods, a new crystal orientation has been chosen such that the incident $\mathrm{x}$-ray beam is again parallel to the hexagonal planes of the crystal but the Ewald sphere intersects the reciprocal lattice differently [as sketched in Fig. 1(b) by the dashed line]. In this case the Ewald sphere misses many rods of low order but does cross the (32l) and (64l) rods. The scattering into these high-order rods is then much weaker, mainly due to the rapid decay of the form factor $F(q)$. Consequently, the probability of multiple scattering via Bragg rods is significantly reduced and the lines of diffraction spots $(00 l)$ and $(96 l), l=$ integer, are clearly visualized and free of scattering between the spots. This observation thus confirms the origin of the secondary Bragg rods in Fig. 2, which arise due to dynamic diffraction via the primary Bragg rods of Fig. 1(a). The estimates given above show that the presence of longrange order along the beam is essential for the transition into the dynamic regime. This intraplanar order is complementary to the interplanar long-range order found earlier [13]. Development of appropriate theory is, however, needed in order to exploit dynamic diffraction for a detailed quantitative structural characterization.

The diffraction intensity along the Bragg rods (32l) and (64l) in Fig. 3 is seen to smoothly vary and display a periodic modulation with minima at integer values of $l$ and broad maxima in between them. This profile of the structure factor along the rod is typical for an rhcp crystal with stacking parameter $\alpha=0.5$. Calculations $[10,13,14,18]$ show that for $\alpha<0.4$ the maxima
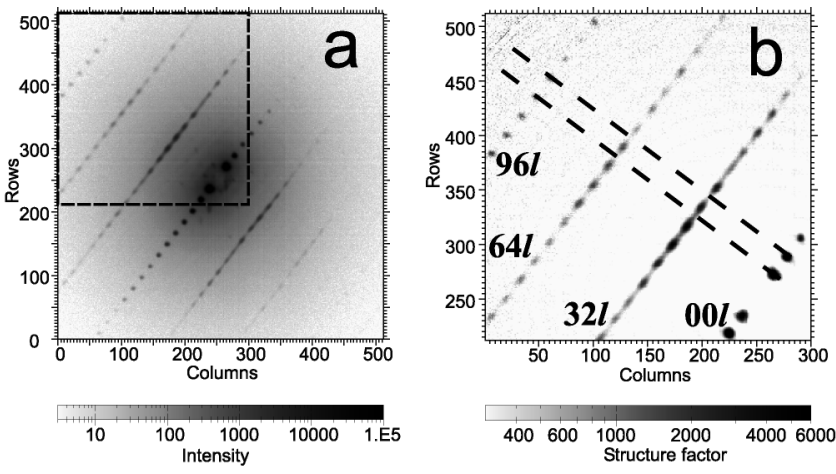

FIG. 3. The same as in Fig. 2 but for another crystal orientation corresponding to the Ewald sphere position shown by the dashed line in Fig. 1(b). The dashed lines in (b) display the position of the shadowed planes of Fig. 1(a) at $l=1$ and $l=2$. 
are narrower and new maxima develop at integer values of $l$. For $\alpha>0.6$ the broad maxima split into two. Note that diffraction into the (001) spots in Fig. 3 is still very strong and might affect the distribution of the $\mathrm{x}$-ray power over the sharp reflections as well as along the rods. However, since this can change the wave vector only by an integer times the $\mathbf{b}_{3}$ vector, these multiple scattering events should not significantly change the intensity profile within one period of the structure factor along the rod, which is equal to $b_{3}$.

In conclusion, this Letter presents the first direct images of Bragg scattering rods in small-angle synchrotron $\mathrm{x}$-ray diffraction obtained from a colloidal single crystal still retaining its random-stacking structure 18 months after crystallization. Moreover, we observed a strong effect of multiple scattering, which reveals itself in the diffraction pattern as secondary Bragg rods. Simple estimates show that, in contrast to common belief, dynamic $\mathrm{x}$-ray diffraction should be rather typical for crystals consisting of highly ordered (sub)micrometer colloidal spheres and has to be taken into account. In our previous work [13], the assignment of the rhcp structure for oneyear-old crystals was based on comparison of intensities of different reflections, which might be somewhat affected by the dynamic diffraction. The present results unambiguously confirm the rhcp structure because multiple scattering cannot broaden sharp reflections into Bragg rods. Since dynamic diffraction is likely to redistribute the diffracted power towards weaker reflections, one should be aware that the amplitude of particle excursions evaluated from the Debye-Waller factor [15] and the spatial extent of the positional order [13] in colloidal crystals could be underestimated.

The authors thank David van der Beek for his assistance in the x-ray diffraction experiment, Alexander Moroz and Wim Bras for useful discussions, and the Netherlands Organisation for the Advancement of Research (NWO) for providing us with the possibility of performing measurements at DUBBLE.

*Corresponding author.

Electronic address: a.v.petukhov@chem.uu.nl

[1] P. N. Pusey and W. van Megen, Nature (London) 320, 340 (1986).

[2] Zh. Cheng et al., Phys. Rev. Lett., 88, 015501 (2002).

[3] Y. A. Vlasov, X.-Z. Bo, J.C. Sturm, and D. J. Norris, Nature (London) 414, 289 (2001); A. Blanco et al., Nature (London) 405, 437 (2000).
[4] A. van Blaaderen, R. Ruel, and P. Wiltzius, Nature (London) 385, 321 (1997).

[5] N. A. M. Verhaegh, J.S. van Duijneveldt, A. van Blaaderen, and H. N.W. Lekkerkerker, J. Chem. Phys. 102, 1416 (1995).

[6] U. Gasser et al., Science 292, 258 (2001).

[7] M. S. Elliot, S. B. Haddon, and W. C. K. Poon, J. Phys. Condens. Matter 13, L553 (2001).

[8] S. I. Henderson and W. van Megen, Phys. Rev. Lett. 80, 877 (1998)

[9] J. Zhu et al., Nature (London) 387, 883 (1997).

[10] Ch. Dux and H. Versmold, Phys. Rev. Lett. 78, 1811 (1997).

[11] W. K. Kegel and J. K. G. Dhont, J. Chem. Phys. 112, 3431 (2000).

[12] W. Vos, M. Megens, C. M. van Kats, and P. Bosecke, Langmuir 13, 6004 (1997); J. E. G. J. Wijnhoven, L. Bechger, and W. L. Vos, Chem. Mater. 13, 4486 (2001).

[13] A. V. Petukhov et al., Phys. Rev. Lett. 88, 208301 (2002).

[14] H. Versmold et al., J. Chem. Phys. 116, 2658 (2002).

[15] M. Megens and W. L. Vos, Phys. Rev. Lett. 86, 4855 (2001).

[16] R.W. James, The Optical Principles of the Diffraction of $X$-Rays (Cornell University Press, Ithaca, NY, 1965); J. M. Cowley, Diffraction Physics (North-Holland, Amsterdam, 1981).

[17] P. G. Bolhuis, D. Frenkel, S.-C. Mau, and D. A. Huse, Nature (London) 388, 235 (1997).

[18] O.S. Edwards and H. Lipson, Proc. R. Soc. London A 180, 268 (1941); A. J. C. Wilson, ibid. 180, 277 (1941); X-Ray Optics (Methuen \& Co. Ltd., London, 1949).

[19] N. A. M. Verhaegh, D. Asnaghi, and H. N.W. Lekkerkerker, Physica (Amsterdam) 264A, 64 (1999); E. H. A. de Hoog et al., Langmuir 17, 5486 (2001).

[20] The extremely small but finite curvature of the Ewald sphere can lead to asymmetry of the diffraction pattern for highly ordered crystals, which are slightly tilted from a low-index orientation [13]. Although we have tried to minimize the tilt angle, some asymmetry can still be seen in Figs. 2 and 3 , which points to the presence of long-range order within hexagonal planes [13].

[21] Q. Shen, in Methods in Materials Research, edited by E. Kaufmann et al. (John Wiley \& Sons, New York, 2000).

[22] V. Yannopapas, N. Stefanou, and A. Modinos, Phys. Rev. Lett., 86, 4811 (2001).

[23] A. Moroz, Phys. Rev. Lett., 83, 5274 (1999).

[24] L. A. Feigin and D. I. Svergun, Structure Analysis by Small-Angle X-Ray and Neutron Scattering (Plenum Press, New York, 1987).

[25] The requirements of crystal quality and the beam coherence are crucial in the longitudinal direction (along the beam) while they are less important in the transverse direction [13]. 